\title{
Presence of Oxytocin, Vasopressin and Atrial Natriuretic Peptide and Their Modification in Rat Hypothalamic Paraventricular Nucleus During Resistance Training
}

\author{
E. V. Farina ${ }^{1 *}$, F. Cappello ${ }^{1}$, L. Lipari ${ }^{2}$, A. Valentino ${ }^{1}$, V. Di Felice ${ }^{1}$ and B. Valentino ${ }^{1}$ \\ Addresses of authors: ${ }^{1}$ Dipartimento di Biomedicina Sperimentale e Neuroscienze Cliniche, Sezione di Anatomia Umana, Università di Palermo, \\ Palermo, Italy; \\ ${ }^{2}$ Dipartimento di Biomedicina Sperimentale e Neuroscienze Cliniche, Sezione di Istologia ed Embriologia, Università di Palermo, Palermo, Italy
}

\author{
*Correspondence: \\ Tel.: +39091 6553510; \\ fax: $+39-091-6553580$ \\ e-mail: farina.elviravitt@tiscali.it
}

With 2 figures

Received August 2012; accepted for publication February 2013

doi: 10.1111/ahe.12051

\begin{abstract}
Summary
Many studies have demonstrated the physiological effects of oxytocin (OT), atrial natriuretic peptide (ANP) and vasopressin (VP) in the homoeostasis of body fluids during physical exercise. However, a little information is available about the related immunohistochemical changes in hypothalamic magnocellular neurosecretory system during and after the training. The aim of the present work was to study the immunohistochemical changes in OT, ANP and VP levels in the hypothalamic paraventricular nucleus during and after resistance exercise protocol. Three groups of Wistar rats were trained by a rung ladder protocol for 15, 30 and 45 days, respectively; a fourth group was left to rest for 15 days after the training. Finally, four sedentary groups were used as controls. The results show that resistance training induces a significant reduction in the percentage of OT-positive neurons, compared with sedentary controls. In contrast, this protocol did not induce any change in VP levels, and ANP levels did not change significantly. However, VP increased after the resting period of 15 days. Our work shows that neurons of the paraventricular nucleus are involved in body fluid homoeostasis during and after resistance exercise. The functional significance of these changes in OT and VP levels, during and after the protocol, needs to be further investigated.
\end{abstract}

\section{Introduction}

Body fluid volume homoeostasis and osmolality are key factors for organism survival (Bourque, 2008; Verbalis, 2010). In different stress conditions, many regulatory mechanisms act simultaneously for the achievement of their maintenance (Morimoto and Itoh, 1998; Cheuvront et al., 2010). Among such stress conditions, there is prolonged physical activity that induces a number of metabolic adjustments aiming to regulate cardiac activity as well as ions and water equilibrium (Maughan, 1992; Donaldson et al., 2003; Maughan and Shirreffs, 2004). Several hormones, such as oxytocin (OT) and vasopressin (VP) - synthesized mainly by the hypothalamic supraoptic (SON) and paraventricular (PVN) nuclei - and atrial natriuretic peptide (ANP) - mostly released by heart and brain - are closely involved in this regulation (Han et al., 1993; Chou et al., 1995a,b).

Previous studies have demonstrated that intense physical activity induces hypoxia in skeletal muscle (Richardson et al., 1995; Hoppeler and Vogt, 2001; Schmutz et al., 2010). Hypoxia, in turn, determines release of OT (Braga et al., 2000) and VP (Stebbins, 1992) in blood of trained rats and cats, respectively, that modulates exercise-induced tachycardia. Similarly, Bonifazi et al. (1994) studied twelve top-level male endurance swimmers and showed that an intense effort taking place during a competition can induce a significant increase in plasma ANP levels that, in turn, exerts an inhibitory effect on plasma rennin activity and aldosterone concentration. However, most of these studies concentrate on endurance trainings, while little information is available about resistance protocols. 
In previous works, we have showed that levels of OT, VP and ANP change in the supraoptic nucleus after resistance protocol in rats (Farina et al., 2008, 2010). The aim of this study was to investigate changes in the levels of these peptides in another hypothalamic nucleus, the paraventricular nucleus (PVN), applying the same training protocol. In our opinion, changes in the expression of these hormones in the hypothalamic nuclei may be associated with their release into the circulation and to the subsequent systemic effects. Hence, our morphologic studies should be considered as preliminary research to be confirmed by further physiological investigation.

\section{Materials and Methods}

\section{Animal care}

Seventy-two-six-month-old male Wistar rats (315.40 \pm 30.30 g body weight) were used in this study in accordance with the Guide for the Care and Use of Laboratory Animals. The rats were divided randomly into eight groups, each including nine animals. Four groups were trained, and four correspondent groups were used as sedentary controls. Animals were housed in cages and were allowed food and water ad libitum. The daily cycle extended from 7 a.m. to 7 p.m., and the room temperature was maintained at $21.6 \pm 0.5^{\circ} \mathrm{C}$. The sedentary animals remained in their cages for the entire duration of the experiments and were sacrificed after 15 (C15), 30 (C30), 45 (C45) and 60 (C60) days, respectively. Animals from three trained groups were sacrificed immediately after the training period, after 15 (T15), 30 (T30) and 45 (T45) days, respectively. Animals from the last trained group were rested for 15 days after the training period before to be sacrificed on day 60 (T60).

\section{Resistance training protocol}

We adopted, with modifications, an already published resistance training protocol (Barone et al., 2009). An exercise consisting in climbing a $1-\mathrm{m}$ ladder with a $2-\mathrm{cm}$ grid and weight attached to the rats' tails was used as resistance training. Rats were familiarized with the exercise for 3 days. The first week after familiarization, the exercise was performed at natural load. To increase the workload, from the second week, increasing weights were attached to the base of the tail with a Velcro strap. The initial weight was $50 \%$ of the rat's body weight and gradually increased throughout the subsequent 6-week training period (on the 30th day: 100\%; on the 45th day: $120 \%)$. The resistance training consisted of 1 set of 10 repetitions with a 1-min rest interval between the repetitions, for 5 days/week. When the rats reached the top of the ladder, they were allowed to recover in the testing area. The training was performed at the same time of the morning each day.

\section{Tissue preparation and immunohistochemistry}

Immediately after the sacrifice, the brain was removed and fixed in Bouin's solution. Successively, the samples were dehydrated in a graded series of alcohol, cleared in xylene and paraffin-embedded. Seven- $\mu \mathrm{m}$ coronal sections of containing the PVN were obtained with a microtome (Leica RM2145), dried overnight at $37^{\circ} \mathrm{C}$ and then stored at room temperature until use. On the day of the experiments, slides were dewaxed in xylene and rehydrated in a graded series of alcohol. Slides were then transferred into distilled water for $5 \mathrm{~min}$.

Immunohistochemistry was performed using the DakoCytomation EnVision + System-HRP kit from Dako (Dako, Glostrup, Denmark), following the manufacturer's instructions. Briefly, sections were covered with the peroxidase block reagent and incubated for $5 \mathrm{~min}$ at room temperature. The samples were rinsed once in PBS buffer ( $\mathrm{pH}$ 7.2). Subsequently, sections were covered with the antibody solution and incubated at $4{ }^{\circ} \mathrm{C}$ overnight. The following primary antibodies were used: rabbit anti-OT polyclonal antibody (1:800 dilution, Cat. No. AB911; Chemicon, Temecula, CA, USA), rabbit anti-VP polyclonal antibody (Chemicon, 1:800 dilution, Cat. No. AB1565) and rabbit anti-ANP polyclonal antibody (Chemicon, 1:800 dilution, Cat. No. AB1970). Each antibody was diluted in a $0.1 \%$ BSA solution. Samples were rinsed twice in PBS pH 7.2 and then incubated with the peroxidase labelled polymer reagent for 30 min. Samples were then rinsed again twice in PBS pH 7.2 and incubated with the substrate-chromogen reagent (AminoEthylCarbazole, AEC). Negative control samples were treated in the same way with primary antibodies substituted with a $0.1 \%$ BSA solution. Slides were coverslipped using the Dako Cytomation Faramount Aqueous Mounting Medium. Experiments were performed in triplicate.

The specimens were observed under a Leica DM1000 light microscope. The percentage of OT, ANP and VP immunopositive neurons in PVN were obtained from all examined specimens by two independent observers (EVF and FC) in 'double-blind'. In detail, the counting of the positive cells was performed inside the PVN for each slide. Results were expressed as percentage of neurons in PVN immunopositive for OT, ANP and VP. The means of obtained values were used as results for the statistical analyses.

\section{Statistical analyses}

Standard statistical analysis was applied to calculate means and standard deviation. A one-way analysis of 
variance (ANOVA) was used to determine the presence of differences between the data obtained by immunostaining. Differences between the means were regarded as significant when a value of $P<0.05$ was obtained.

\section{Results}

We found that resistance training reduces the quantity of OT in the PVN. In particular, the T15, T30 and T45 groups showed a significant reduction in the percentage of positive neurons compared with sedentary controls (Fig. 1a). Interestingly, the T60 group did not show any
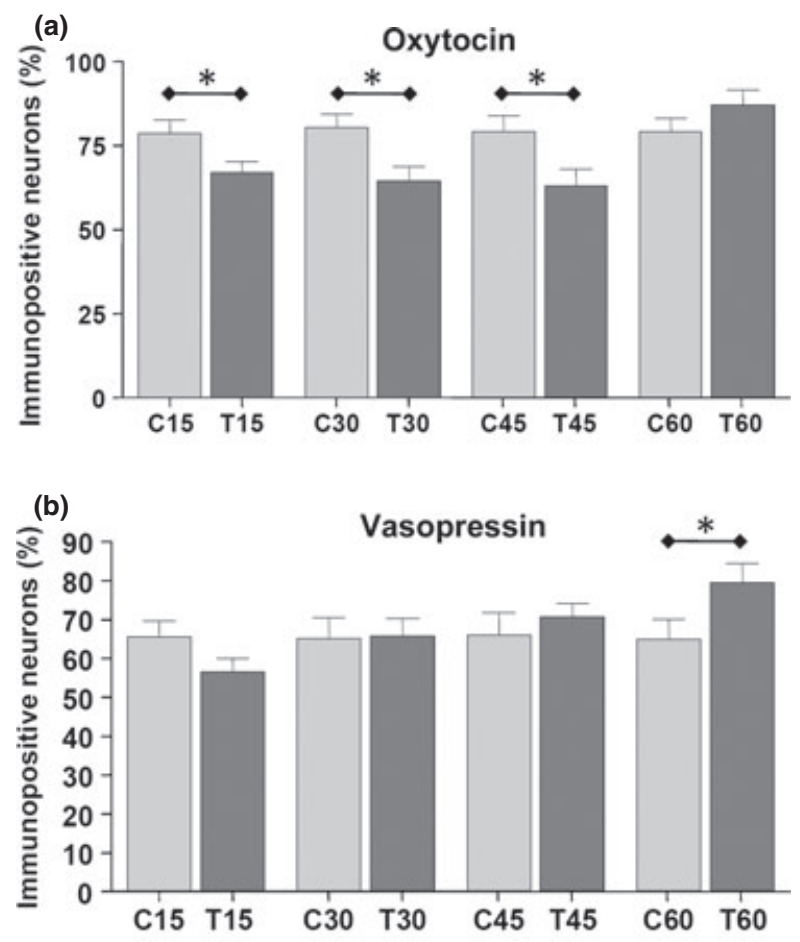

(c)

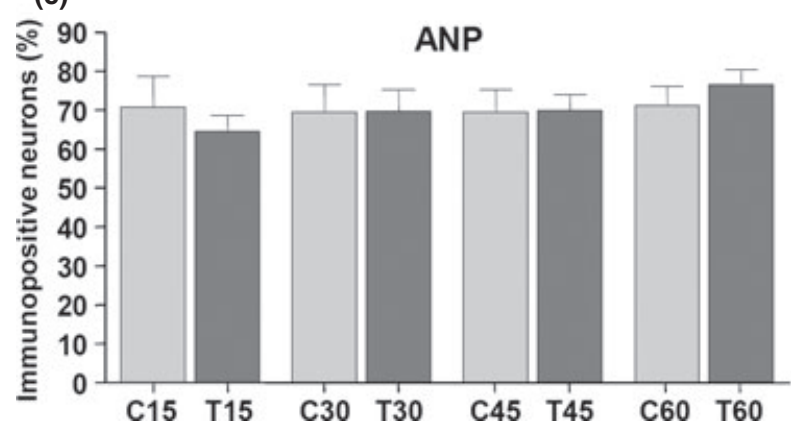

Fig. 1. These histograms show the results of statistical analyses. In particular, the percentage of positive neurons for oxytocin, vasopressin and ANP in PVN was compared in trained ( $\mathrm{T}$ ) and sedentary (C) animals. Asterisks show the presence of a significant difference. See the text for further details. significant difference compared with C60. Hence, we postulate that a resting period of 15 days is enough to determine a recovery of OT in PVN neurons. Figure 2 shows representative pictures of the OT immunostainings.

With regard to VP expression, we did not find any significant differences in the percentage of PVN-positive neurons between the T15, T30 and T45 groups and sedentary controls (Fig. 1b). In contrast, we found a significant increase in the percentage of positive cells in the T60 group compared with C60. Hence, we hypothesize that a 15-days resting period after 45 days of training may induce an increase in VP positivity. Figure 2 shows representative pictures of the VP immunostainings.

Finally, we did not find any significant differences in the percentage of ANP-positive neurons between trained and sedentary groups (Fig. 1c).

\section{Discussion}

Our experimental resistance protocol induced changes in the levels of OT and VP but not of ANP. In particular, training significantly reduced the percentage of neurons positive for OT, but after a resting period of 15 days, OT levels recovered to control levels. These results indicate that OT levels in PVN are sensible to resistance training protocol. We postulate that these changes may be related to either a reduced production or an increased secretion of this hormone; both mechanisms are not mutually exclusive and may have regulatory effects on body fluid homoeostasis.

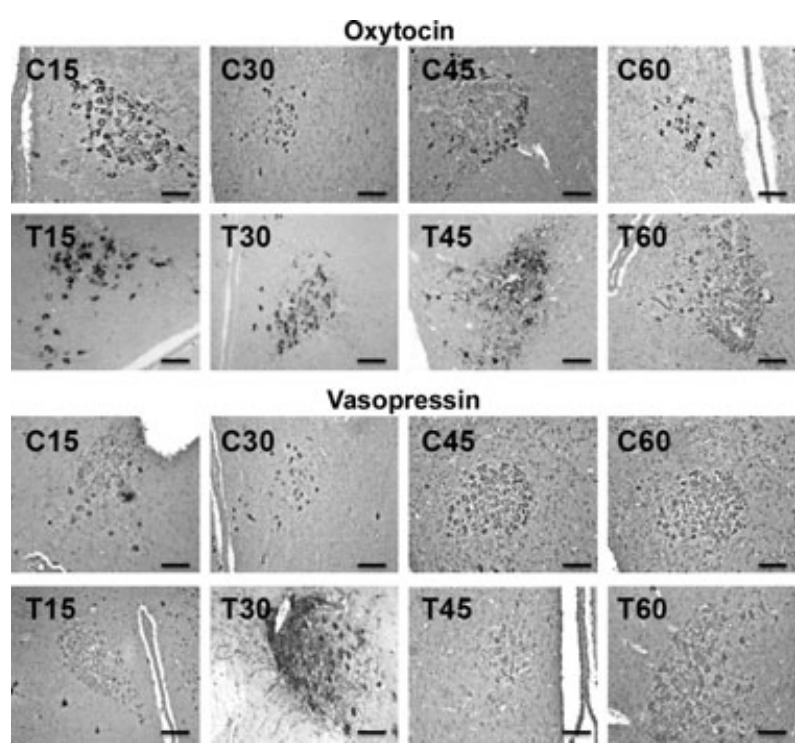

Fig. 2. This panel shows representative pictures of PVN sections immunostained for oxytocin and vasopressin in all examined groups. Bar: 100 micra. 
In contrast, we found that our protocol did not induce any changes in VP levels. Interestingly, after the resting period, VP expression increased significantly in the trained groups compared with controls. In our opinion, this means that VP may contribute to post-training adaptive changes aimed to restore the general homoeostasis of the organism.

Finally, ANP levels in the PVN did not change significantly. However, this does not exclude that ANP, that is also secreted by other organs, such as the heart and nuclei, as supraoptic nucleus, affects homoeostatic control during resistance exercise (Farina et al., 2010).

Our results on OT and VP expression are partially in agreement with previous studies showing that these hormones play a role in controlling body fluid volume homoeostasis and osmolality during exercise and physical stress (Stebbins, 1992; Bonifazi et al., 1994; Dufloth et al., 1997; Li et al., 1997; Braga et al., 2000; Michelini, 2001; Neumann et al., 2006; Higa-Taniguchi et al., 2009). In particular, Neumann et al. (2006) demonstrated that forced swimming in rats determines both OT and VP release by PVN. However, only OT was found to have systemic effects, being responsible for an ACTH response. Michelini (2001) and Dufloth et al. (1997) showed that other encephalic nuclei, such as the nucleus tractus solitarii (NTS) and the dorsal motor nucleus of the vagus, participate in OT and VP release during exercise.

Besides the effects on vascular tone, renal function and, in turn, volaemia (Verbalis et al., 1991; Petersson et al., 1999), OT has also been shown to affect central autonomic functions. Indeed, OT neurons projections arising from PVN may reach brainstem areas controlling cardiovascular function (Buijs, 1978; Sofroniew and Schrell, 1981; Sawchenko and Swanson, 1982). Higa-Taniguchi et al. (2009) demonstrated that exercise training was also effective in augmenting OT expression in NTS and increasing exercise tachycardia in hypertensive rats after NTS OT receptor blockade. Our data show that OT levels in PVN reduce during exercise. Takes together, these data may indicate that there is an equilibrium between NTS and PVN in producing OT and that this equilibrium may be crucial in regulating cardiovascular functions during exercise. Whether VP produced by PVN participates in this equilibrium needs to be proved. However, we may postulate that these hormones may constitute a part of the neural pathway involved in cardiovascular regulation during exercise.

In conclusion, our results show that PVN has an intensive activity during and after resistance exercise, as already showed for endurance activity (Barna et al., 2012). Our work also supports the hypothesis that OT and VP are involved in body fluid homoeostasis during and after resistance exercise. Further studies are necessary to investigate their effects in distant organs, such as heart or kidney.

\section{References}

Barna, B. F., A. C. Takanara, and T. S. Moreira, 2012: Pontomedullary and hypothalamic distribution of Fos-like immunoreactive neurons after acute exercise in rats. Neuroscience 212, 120-130.

Barone, R., M. Bellafiore, V. Leonardi, and G. Zummo, 2009: Structural analysis of rat patellar tendon in response to resistance and endurance training. Scand. J. Sci. Sports 19, 782-789.

Bonifazi, M., E. Bela, G. Carli, L. Lodi, C. Lupo, E. Maioli, G. Martelli, and A. Viti, 1994: Responses of atrial natriuretic peptide and other fluid regulating hormones to long distance swimming in the sea. Eur. J. Appl. Physiol. Occup. Physiol. 68, 504-507.

Bourque, C. W., 2008: Central mechanisms of osmosensation and systemic osmoregulation. Nat. Rev. Neurosci. 9, 519-531.

Braga, D. C., E. Mori, K. T. Higa, M. Morris, and L. C. Michelini, 2000: Central oxytocin modulates exerciseinduced tachycardia. Am. J. Physiol. Regul. Integr. Comp. Physiol. 278, R1474-R1482.

Buijs, R. M., 1978: Intra- and extrahypothalamic vasopressin and oxytocin pathways in the rat. Pathways to the limbic system, medulla oblongata and spinal cord. Cell Tissue Res. 192, 423-435.

Cheuvront, S. N., R. W. Kenefick, S. J. Montain, and M. N. Sawka, 2010: Mechanisms of aerobic performance impairment with heat stress and dehydration. J. Appl. Physiol. 109, 1989-1995.

Chou, C. L., S. R. Di Giovanni, R. Mejia, S. Nielsen, and M. A. Knepper, 1995a: Oxytocin as an antidiuretic hormone. I. Concentration dependence of action. Am. J. Physiol. 269, F70-F77.

Chou, C. L., S. R. Di Giovanni, A. Luther, S. J. Lolait, and M. A. Knepper, 1995b: Oxytocin as an antidiuretic hormone. II. Role of V2 vasopressin receptor. Am. J. Physiol. 269, F78-F85.

Donaldson, G. C., W. R. Keatinge, and R. D. Saunders, 2003: Cardiovascular responses to heat stress and their adverse consequences in healthy and vulnerable human populations. Int. J. Hyperthermia 19, 225-235.

Dufloth, D. L., M. Morris, and L. C. Michelini, 1997: Modulation of exercise tachycardia by vasopressin in the nucleus tractus solitarii. Am. J. Physiol. 273, R1271-R1282.

Farina, E. V., A. Lipari, M. Bellafiore, G. Peri, A. Gerbino, M. Buscemi, and B. Valentino, 2008: The neurosecretion of oxytocin in the hypothalamic magnocellular nuclei of training rats. Folia Hystochem. Cytobiol. 46, S111-S112.

Farina, E. V., D. Lipari, and B. Valentino, 2010: Modifications of atrial natriuretic peptide and vasopressin peptides in rat hypothalamic supraoptic nucleus during resistance training. Ital. J. Ant. Embryol. 115, 211-217.

Han, J. S., Y. Maeda, and M. A. Knepper, 1993: Dual actions of vasopressin and oxytocin in regulation of water permeability in terminal collecting duct. Am. J. Physiol. 265, F26-F34. 
Higa-Taniguchi, K. T., J. V. Felix, and L. C. Michelini, 2009: Brainstem oxytocinergic modulation of heart rate control in rats: effects of hypertension and exercise training. Exp. Physiol. 94, 1103-1113.

Hoppeler, H., and M. Vogt, 2001: Muscle tissue adaptations to hypoxia. J. Exp. Biol. 204, 3133-3139.

Li, J., G. A. Hand, J. T. Potts, and J. H. Mitchell, 1997: Identification of hypothalamic vasopressin and oxytocin neurons activated during the exercise pressor reflex in cats. Brain Res. 752, 45-51.

Maughan, R. J., 1992: Fluid balance and exercise. Int. J. Sports Med. 13(Suppl. 1), S132-S135.

Maughan, R., and S. Shirreffs, 2004: Exercise in the heat: challenges and opportunities. J. Sports Sci. 22, 917-927.

Michelini, L. C., 2001: Oxytocin in the NTS. A new modulator of cardiovascular control during exercise. Ann. N. Y. Acad. Sci. 940, 206-220.

Morimoto, T., and T. Itoh, 1998: Thermoregulation and body fluid osmolality. J. Basic Clin. Physiol. Pharmacol. 9, 51-72.

Neumann, I. D., L. Torner, N. Toschi, and A. H. Veenema, 2006: Oxytocin actions within the supraoptic and paraventricular nuclei: differential effects on peripheral and intranuclear vasopressin release. Am. J. Physiol. Regul. Integ. Comp. Physiol. 291, R29-R36.

Petersson, M., T. Lundeberg, and K. Uvnäs-Moberg, 1999: Short-term increase and long-term decrease of blood pressure in response to oxytocin-potentiating effect of female steroid hormones. J. Cardiovasc. Pharmacol. 33, 102-108.
Richardson, R. S., D. R. Knight, D. C. Poole, S. S. Kurdak, M. C. Hogan, B. Grassi, and P. D. Wagner, 1995: Determinants of maximal exercise VO2 during single leg knee-extensor exercise in humans. Am. J. Physiol. 268, H1453-H1461.

Sawchenko, P. E., and L. W. Swanson, 1982: Immunohistochemical identification of neurons in the paraventricular nucleus of the hypothalamus that project to the medulla or to the spinal cord in the rat. J. Comp. Neurol. 205, 260-272.

Schmutz, S., C. Däpp, M. Wittwer, A. C. Duriex, M. Mueller, F. Weinstein, M. Vogt, H. Hoppeler, and M. Flück, 2010: A hypoxia complement differentiates the muscle response to endurance exercise. Exp. Physiol. 95, 723-735.

Sofroniew, M. V., and U. Schrell, 1981: Evidence for a direct projection from oxytocin and vasopressin neurons in the hypothalamic paraventricular nucleus to the medulla oblongata: immunohistochemical visualization of both the horseradish peroxidase transported and the peptide produced by the same neuron. Neurosci. Lett. 22, 211-217.

Stebbins, C. L., 1992: Reflex cardiovascular response to exercise is modulated by circulating vasopressin. Am. J. Physiol. 263, R1104-R1109.

Verbalis, J. G., 2010: Brain volume regulation in response to changes in osmolality. Neuroscience 168, 862-870.

Verbalis, J. G., M. P. Mangione, and E. M. Stricker, 1991: Oxytocin produces natriuresis in rats at physiological plasma concentrations. Endocrinology 128, 1317-1322. 